\title{
El papel de la familia en la construcción de la competencia digital
}

\author{
Esther Martínez-Piñeiro ${ }^{1}$, Esther Vila Couñago ${ }^{1}$, Adriana Gewerc Barujel ${ }^{1}$ \\ esther.martinez@usc.es, esther.vila@usc.es, adriana.gewerc@usc.es \\ ${ }^{1}$ Facultad de Ciencias de la Educación, Campus Vida, 15782, Santiago de Compostela, España.
}

DOI: 10.17013/risti.28.1-13

\begin{abstract}
Resumen: La competencia digital de los jóvenes se desarrolla más allá de la escuela; familia y amigos son agentes de aprendizaje. A través de un estudio de casos, financiado por el Ministerio de Economía y Competitividad español, se estudió el papel de la familia en la construcción de la competencia digital de niños/ as de doce años en hogares con distinto capital cultural. Destacamos el distinto uso y significado de la tecnología: en familias de capital cultural alto ocupan un papel secundario -en función del valor y el tiempo que le dedican-, ya que su status no depende del dominio de las tecnologías; en las familias de nivel bajo son vistas como medio de acceso a la información y al conocimiento y apenas hay normas que limiten su empleo, y en las de nivel medio - con pautas que las regulan- son percibidas como instrumento para el ascenso social.
\end{abstract}

Palabras-clave: competencia digital; capital cultural.

\section{The role of the family in building digital competence}

\begin{abstract}
Young people's digital competence develops beyond school; family and friends are learning agents. A study was carried out on the role of the family in building the digital competence of twelve-year-old children in households with different cultural capital, through case studies, funded by the Spanish Ministry of Economy and Competitiveness. We highlight the different use and meaning of technology: in families with high cultural capital they occupy a secondary role -in the value they attach to it and the time they devote to it-, since their status does not depend on the mastery of ICTs; in low-level families they are seen as a means of access to information and knowledge and there are hardly any rules that limit their use, and in mid-level families -with guidelines that regulate them- they are perceived as an instrument for social advancement.
\end{abstract}

Keywords: digital competence, cultural capital

\section{Introducción}

Las tecnologías digitales se han hecho imprescindibles en la vida cotidiana, tanto en el plano personal, como social y laboral; no sólo facilitan el acceso a recursos e información, 
sino que en muchas ocasiones son ya el único medio para llegar a ellos. En esta línea la OCDE (2014) prevé que seguirán siendo un motor clave en la creación de empleo, y asume el desarrollo de competencias para el uso adecuado de las Tecnologías de la Información y la Comunicación (TIC) como la más importante estrategia para las políticas de recuperación económica. Validando esta importancia, más recientemente, en enero de 2018, la Comisión Europea lanza el Plan de Acción de Educación Digital que abarca 3 prioridades principales con 11 acciones para fomentar y apoyar el uso de la tecnología en educación, así como el desarrollo de la competencia digital. En ese contexto, la preocupación por la inclusión digital está vigente, ya que las tecnologías digitales posibilitan la participación plena de la ciudadanía en numerosos procesos y acciones, en definitiva, favorecen una completa inclusión social de los sujetos. Tener escasa competencia digital dificulta el acceso a la cultura, el empleo y al ejercicio de los derechos, por eso el binomio inclusión digital-inclusión social cobra relevancia en esta realidad.

Se hace necesaria, por lo tanto, formación en las competencias y habilidades que permitan un uso apropiado y pertinente de las tecnologías digitales desde los primeros años de escolaridad, que capacite al alumnado a enfrentarse y adaptarse a los acelerados cambios que el desarrollo tecnológico impulsa en la sociedad contemporánea (Colette \& da Silva, 2014).

Pero niños/as y adolescentes aprenden no sólo en el seno de la escuela, a lo largo de su educación formal. Los amigos, la familia o los vecinos, pueden ser también agentes formativos, y el ocio u otras actividades sociales pueden constituir espacios para el aprendizaje.

En este contexto, presentamos algunos de los resultados del proyecto CDEPI "Competencia digital en estudiantes de educación obligatoria. Entornos socio-familiares, procesos de apropiación y propuestas de inclusión”, que está llevando a cabo el grupo de investigación Stellae, de la Universidad de Santiago de Compostela en España (financiado por el Ministerio de Economía y Competitividad-Fondos FEDER, EDU2015-67975-C31-P). A continuación, se presenta el marco teórico y metodológico de la investigación, los resultados y discusión acerca del papel de la familia en la apropiación de la competencia digital y las principales conclusiones al respecto.

\section{Marco teórico}

Este proyecto pretende indagar en la competencia digital del alumnado de sexto curso de educación primaria -último curso de esta etapa- con la intención de conocer el proceso de aprendizaje de la competencia digital y el papel que juegan en él la familia y el grupo de iguales, así como la influencia de este aprendizaje en los procesos de inclusión social -entendida, desde términos comúnmente aceptados, como posibilidad efectiva de participación de todas las personas en todas las dimensiones sociales propias de una ciudadanía activa-.

Dos son los ejes centrales en el marco teórico-conceptual de partida: 1 . el concepto de competencia digital, 2. la relación capital cultural-apropiación de la tecnología.

\subsection{El concepto de competencia digital}

Las habilidades y competencias digitales implican saberes y procesos complejos. Estas se conocen como alfabetización digital (Area, 2012), en el mundo angloparlante, digital 
literacy o digital literacies (Lankshear \& Knobel, 2008), o también como alfabetización o competencia mediática (Buckingham, 2007), habilidades digitales (Van Dijk \& Van Deursen, 2014) y nuevos alfabetismos (Dussel, 2009).

El proyecto del Marco Europeo de Competencia Digital (también conocido como DIGCOMP) (Ferrari, 2013; Vuorikari, Punie, Carretero \& Van den Brande, 2016) desarrolla un modelo conceptual de la competencia digital, en el que se identifican sus dimensiones o indicadores, que hemos tomado como referencia en nuestro estudio. En concreto la competencia digital es definida como:

un conjunto de conocimientos, habilidades, actitudes, estrategias y valores que son puestos en acción cuando usamos las tecnologías y los medios digitales para realizar tareas, resolver problemas, comunicarse, tratar información, colaborar, crear y compartir contenidos y crear conocimiento, de forma efectiva, eficiente, apropiada, crítica, creativa, autónoma, flexible, ética y reflexiva para el trabajo, el ocio, la participación, el aprendizaje, la socialización, el consumo y el empoderamiento (Ferrari, 2013, p. 30).

DIGCOMP desarrolla un modelo conceptual con cinco dimensiones (Información, Comunicación, Creación de Contenidos, Seguridad y Resolución de problemas) y 21 subcompetencias. En cinco años de vigencia el modelo se ha aplicado a diferentes ámbitos (educación, formación y empleo) con diversos usos (políticas de apoyo; planificación de la educación; evaluación y acreditación).

\subsection{Capital cultural y procesos de apropiación de la tecnología}

En estos momentos, sobre la competencia digital pivotan aspectos fundamentales que determinan la frontera entre inclusión y exclusión, además de los ya evidentes que actúan sobre colectivos excluidos o en riesgo de exclusión: nivel socioeconómico, cultura(s), etc. Para comprender los patrones de exclusión social en este periodo histórico, necesitamos saber cómo los niños y la tecnología interactúan y cómo se transforman entre sí (Bijker \& Law, 1992).

Visualizar estas prácticas situadas en el ambiente socio-familiar podría ser determinante para que se identifiquen diferencias significativas en los modos de relacionarse con la tecnología (Hollingworth, Mansaray, Allen \& Rose, 2011). Para esto, los conceptos de habitus, campo y capital de Pierre Bourdieu ayudan a comprender cómo las posiciones en los campos sociales y culturales de las familias y las estrategias que emplean, limitan o posibilitan el desarrollo de la competencia digital de los niños, en la línea que ya han planteado investigaciones previas (Johnson 2009a, 2009b). El concepto de habitus se utilizó para estudiar la relación entre jóvenes, gustos digitales y la clase social de pertenencia (North, Snyder, \& Bulfin, 2008).

El habitus es definido por Gutiérrez (2005, p. 68) como "aquellas disposiciones a actuar, percibir, valorar, sentir y pensar de una cierta manera más que de otra, disposiciones que han sido interiorizadas por el individuo en el curso de su historia”. El capital cultural es el potencial adquirido de la cultura intelectual y del medio familiar. Son las formas de conocimiento, educación, habilidades y ventajas que tiene una persona y que le dan un status más alto dentro de la sociedad (Bourdieu, 2002). 
Las prácticas con las tecnologías no son neutrales, no tienen el mismo significado para todos, y no pueden ser analizadas de forma aislada sin tener en cuenta el entorno de cada de cada niño/a. Su uso se integra en el estilo de vida y en los esquemas familiares y son apropiadas de forma diferente. Los recursos que poseen las familias, su empleo y pautas de uso están relacionados con su habitus y éstos con el capital cultural que poseen.

\section{Metodología}

El estudio está centrado en el estudiantado de $6^{\circ}$ curso de centros públicos de Educación Primaria de Galicia, Madrid y Castilla la Mancha. En este artículo nos centraremos en el diseño específico empleado en los casos de la comunidad gallega y en los resultados obtenidos en parte de los mismos.

Para conseguir los objetivos propuestos se optó por un diseño mixto en el que el método principal es el estudio de casos múltiple, de corte cualitativo. Creswell y Plano Clark (2007) definen la investigación con métodos mixtos como un procedimiento para recoger, analizar y combinar métodos tanto cuantitativos como cualitativos en el marco del mismo estudio o en una serie de estudios para comprender un problema de investigación.

Nuestro diseño se encuadra dentro de los diseños secuenciales explicativos, aquellos en los que en la primera parte se emplea un método cuantitativo cuyos resultados son necesarios para llevar a cabo la segunda fase, cualitativa, en nuestro caso la principal del estudio (Creswell \& Plano Clark, 2007). Además, dentro de este tipo de diseños, atendiendo a la función que cumple el estudio cuantitativo, estamos ante un diseño explicativo llamado de "selección de participantes". Este es usado cuando el investigador necesita información cuantitativa para seleccionar los participantes en el estudio cualitativo. Tal y como se representa en la Figura 1, en una primera fase se recogieron datos de una muestra amplia de sujetos utilizando el método de encuesta. A partir de los resultados obtenidos, se seleccionaron los sujetos que participaron en el estudio de casos. Presentamos con más detalle cada una de las fases.

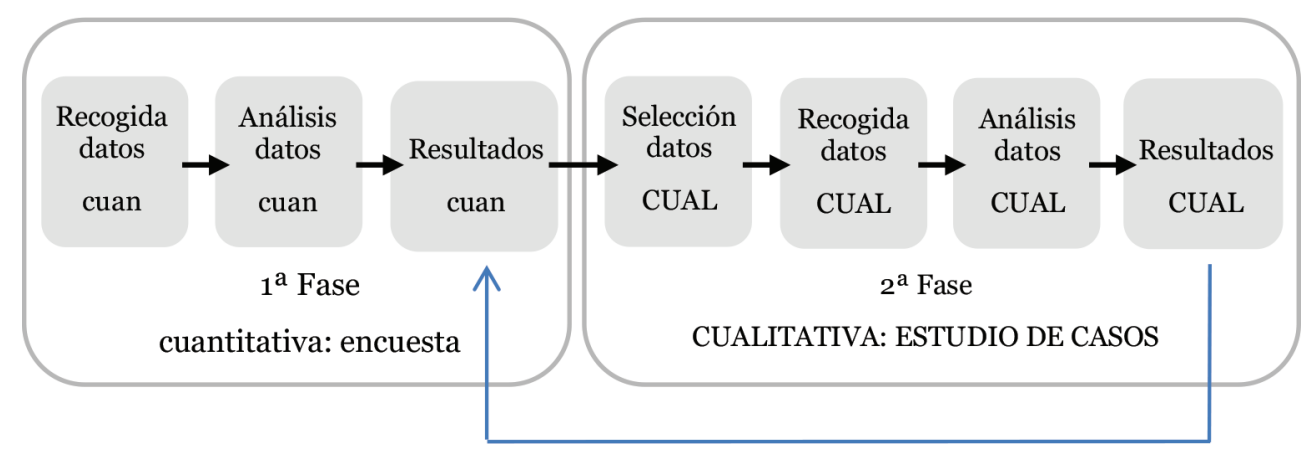

Figura 1 - Diseño mixto explicativo de selección de participantes

\subsection{Primera fase: selección de los casos}

Para la selección de los sujetos que conformarían nuestros casos de estudio nos dirigimos inicialmente a centros educativos inmersos en proyectos de saturación tecnológica, 
en los que se aplicó un cuestionario dirigido a las familias del alumnado de sexto de primaria con el objetivo de seleccionar posibles casos de estudio atendiendo a los siguientes criterios:

- Representantes de entornos socio-culturales y económicos diferentes (capital cultural bajo, medio y alto)

- Niños y niñas, atendiendo a la importancia que puede tener el género en este proceso de construcción de la competencia digital.

- Que se presten a la investigación (criterio de accesibilidad).

A través de este instrumento pretendíamos conocer algunas condiciones del entorno socio-familiar que permitiese seleccionar casos según el capital cultural, a partir de determinados indicadores (dispositivos tecnológicos existentes, estudios y profesión de los padres, y concesión de ayudas y bolsas, fundamentalmente).

El instrumento fue previamente validado por un conjunto de expertos en TIC y en metodología de investigación.

El cuestionario consta de cuatro partes: la primera relativa a las características sociodemográficas del niño/a de sexto curso; la segunda sobre los usos de la tecnología en el hogar; la tercera sobre los hábitos del niño/a; y una cuarta sobre datos familiares, donde se incluían las cuestiones relativas a la ocupación y estudios de los padres, solicitud de ayudas, etc.

Se recogió información de un total de 182 familias con niños de cinco centros educativos diferentes. Tras el análisis de las respuestas dadas a los ítems relevantes, se preseleccionaron un grupo de niños y niñas pertenecientes a distintos centros y a entornos familiares con distinto capital cultural. Desde el centro educativo se comunicó a la familia esta selección invitándole a su participación.

\subsection{Segunda fase: Estudio de casos como método principal}

Fueron seleccionados finalmente un total de seis sujetos en la comunidad gallega, que conformaron el estudio de casos múltiple: dos pertenecientes a familias de capital cultural alto (dos hermanas), dos de medio (dos hermanos) y dos de bajo (dos niños). Se optó por el método de estudio de casos ya que éste "investiga un fenómeno contemporáneo dentro de su contexto real de existencia, cuando los límites y el contexto no son claramente evidentes" (Yin, 2003, p. 23).

Las técnicas de recogida de datos utilizadas fueron diversas y aplicadas a informantes diferentes, que nos permitieran obtener información rica y compleja y favorecer la triangulación entre fuentes y técnicas. En concreto empleamos:

- Entrevistas en profundidad a los niños/as, padres y madres o tutores según los casos; amigos cercanos, y tutores de la escuela.

- Observación participante de los sujetos del caso: fundamentalmente de los comportamientos del niño/a en su actuación ante las tecnologías. En estas sesiones los sujetos realizaron tareas o jugaron a distintas aplicaciones con su ordenador portátil o su tableta que fueron grabadas para su análisis posterior.

- Diarios de campo del entrevistador/a. 
- Observación no participante del entorno familiar y escolar.

- Diarios de los niños y niñas. Cada caso elaboró un diario en donde recopiló las actividades con tecnologías que realiza en el día a día. Ellos podían elegir el formato apropiado (audio-texto-imagen fija o video) y también el medio por el cual se le enviaba al investigador/a.

- Análisis de documentos del centro escolar al que asisten los niños/as.

La información recogida se analizó atendiendo a las pautas de la teoría fundamentada propuesta por Glaser y Strauss (1967). Se realizó una primera codificación del material recogido de cada uno de los casos, la codificación abierta, en la que el texto fue dividido en porciones de información con significado a los que se les asignó un código, utilizando un procedimiento inductivo. Posteriormente los códigos fueron agrupados en categorías, cuando presentaban un significado similar (Soãres de Lima et al., 2010).

En una segunda fase, se llevó a cabo la codificación axial. En este proceso se establecieron relaciones entre categorías y subcategorías, intentando encontrar claves en el texto que permitieran relacionar las principales categorías entre sí. Este proceso se llevó a cabo empleando el programa Atlas.ti, que permitió no sólo la organización, almacenamiento, etiquetaje y recuperación de la información, sino también el establecimiento de relaciones entre códigos, y la visualización de dichas relaciones en mapas (Muñoz \& Sahagún, 2017).

\section{La familia en el proceso de construcción de la competencia digital}

El análisis de los casos -en concreto nos centramos en los dos de nivel alto, Lucía y Catarina, los dos de nivel medio, Alfonso y Antón, y uno de los casos de nivel bajo, Jaime ${ }^{1-}$ nos ha permitido descubrir el distinto significado social que las tecnologías pueden tener en el seno de la familia, indagando en lo que se considera apropiado, pertinente o por el contrario impensable o fuera de lugar. Este habitus tecnológico influye en la manera en que imponen reglas para su uso, y en cómo se enfrentan a los diferentes aspectos relativos a la competencia digital.

En esta línea, algunas investigaciones llaman la atención sobre el impacto del estilo parental en el uso que los/as niños/as realizan con internet. Así, Valcke, Bonte, Wever y Rots (2010) consideran que el comportamiento de los padres en la red y su nivel educativo son las variables más influyentes en el uso de internet de los menores en el hogar. De este modo, aspectos como el establecimiento de normas, tiempos de uso, o restricción de acceso a determinadas páginas web, condiciona en gran medida la experiencia que los/as niños/as desarrollan en la red. Se hace por ello necesario indagar en la percepción y experiencias de la familia con respecto al uso de las TIC, con el objetivo de poder comprender la naturaleza de las interacciones de los menores.

Ofrecemos en primer lugar una síntesis del sentido que le otorgan a las tecnologías en los distintos hogares atendiendo al capital cultural y de las normas existentes en la familia relativas a su uso.

\footnotetext{
${ }^{1}$ Son todos ellos nombres ficticios que permiten identificar los fragmentos de las trascripciones que incluimos.
} 


\subsection{El papel de la tecnología en el hogar}

Los resultados obtenidos tras los estudios de caso ponen de manifiesto que en los hogares con un capital cultural alto (con mayor destreza tecnológica o no) la tecnología ocupa un papel secundario tanto en sus usos y como en el esquema de valores familiares.

Aunque los dispositivos tecnológicos son cotidianos para las niñas y en su casa hay una diversidad de ellos (varios ordenadores, iPad y consolas), estos no son un elemento relevante en su día a día, si pensamos en el tiempo que le dedican y en la atención que les prestan. Salvo en el caso del móvil, en alguna ocasión, ambas niñas no parecen mostrar inicialmente especial interés por usarlos, por poder contar con más tiempo para ellos o por dotarse de más juegos o dispositivos.

Entrevistadora: ¿No pides usarlo un poquito más?

Lucía: No.

Entrevistadora: No, no te... No te apetece o... ¿No?

Lucía: Tampoco, es que llego tarde de las actividades, entonces... (Entrevista2, Lucía)

Entrevistadora: Ya lo sabéis ¿Y cómo...? ¿Cómo lo ves tú? ¿Te gustaría tener más tiempo para jugar?

Catarina: No, además las utilizo, pero los fines de semanas. Por la semana no tengo tiempo porque tengo muchas actividades, tengo pocos deberes, mi profe no manda casi ningún deber. Tengo que estudiar un instrumento, aunque eso es solo los fines de semana más o menos, y... (Entrevista1, Catarina)

Los padres otorgan mayor importancia a actividades artísticas o de ocio compartido. El esfuerzo, la formación musical, los hábitos saludables, la familia... están en primer lugar. La competencia tecnológica no es una prioridad, pues no se considera necesaria. Ambas niñas están familiarizadas con el ordenador y la tableta desde pequeñas y se manejan con soltura en distintas aplicaciones que utilizan para tareas escolares y, en menor medida, para el ocio. Han ido aprendiendo a utilizarlos en el seno de la familia, y después en la escuela.

Los padres, valoran más la oportunidad de poder interactuar y jugar al aire libre con otros niños y niñas que el uso de dispositivos digitales:

Padre: Claro, ahora, yo tengo mi impresión de que a lo mejor aquí, (...) puede haber más niños... Que los puedes ver más con teléfonos o las tablets, pero es que donde vivimos los niños cuando salen afuera pueden hacer otras cosas, y como de momento todavía salen, tienen a donde salir. Pues se les abre otro abanico.

Entrevistador: Pero eso ¿Lo ves como una oportunidad?

Padre: Claro. Como algo bueno, algo bueno. (Entrevista2, padres Catarina y Lucía)

En los hogares con un capital cultural medio la posesión y uso de dispositivos tecnológicos se ve como una posibilidad para ascender en el status social, como una vía de acceso a mejores posiciones en la escala social. La competencia digital en estos casos es entendida como necesaria, como un medio importante para la movilidad social de sus hijos. Al mismo tiempo como algo que los niños/as necesitan y deben adquirir para participar 
legítimamente en la educación y el aprendizaje y ven la tecnología como un elemento necesario para situarse en este mundo.

Madre: Y... Y después a ver, yo creo que para ellos... que ojo, aparte, ten en cuenta que, a ver... El mundo laboral en el que se van a desenvolver ellos va a ser todo con... informática. (Entrevista1, madre Alfonso y Antón)

A pesar de ser conscientes de los peligros y los riesgos que los medios de comunicación están comentando con respecto a las tecnologías, a la hora de otorgar permisos o de proponer actividades, ponen el acento más en sus beneficios, ya que esto podría ayudar a los niños a acrecentar su capital cultural (Livingstone \& Selfton Green, 2016). En ese contexto, la concepción de la familia es que se acumula capital tanto jugando, o conversando on-line, como haciendo las tareas de la escuela.

Madre: Sí, el curso pasado... Claro, me decían... "Mamá, podemos tal...?” Y yo, "mira, total andáis con el ordenador... bueno, vale. Lo que sí, procurad no poner fotos vuestras..." De momento... Tienen Facebook, tienen Instagram... E Instagram, el curso pasado sobre todo hablaban con muchos críos de afuera... de Estados Unidos y tal, porque estaban en un grupo que les gustaba "The Walking Death" a todos... (Entrevista1, madre Alfonso y Antón)

Tanto los niños como la familia piensan que acercarse a la tecnología (aunque sea por diferentes motivos) implica una forma de aprender, de desarrollar habilidades que son necesarias e importantes para el futuro, quizás más importantes que lo que puedan aprender en la escuela. Desde pequeños se les estimula en el uso de las tecnologías.

Madre: No sabían escribir y te decían a ti: "ponme esto". Y claro no sabían escribir. Pero sabían, quiero decir, no tenían ningún tipo de problema, y tal. (Entrevista1, madre Alfonso y Antón)

En entornos donde los padres ocupan posiciones más bajas en el espacio social constatamos que el uso de la tecnología no está restringido a momentos determinados y atendiendo a la realización o no de otras actividades. Son escasas las restricciones y las pautas familiares establecidas para su empleo, ya que se asume la consideración del uso de la tecnología como una práctica beneficiosa, como un medio de acceso a la información y al conocimiento, tal como podemos ver en los dos fragmentos de entrevista siguientes:

Madre: Claro, y después por ejemplo él me decía: "mamá, es que conocí a un niño mejicano y me dijo que en su país esto es así, así, así”. Claro, y él va cogiendo conocimientos, entonces, ¿por qué le voy a restringir eso? Pues prefiero que este, a lo mejor, hablando online con esos niños que le pueden aportar algo y no viendo... Shin Chan en la tele. (Entrevista1, madre Jaime)

Madre: No... Yo no... Yo ya lo dije, no sé si está bien o está mal, pero yo no le restrinjo para eso, yo creo que... Yo sé en todo momento lo que mi hijo está haciendo con la tablet, y si a mi hijo no le va perjudicar y al mismo tiempo está... Prestando atención y está aprendiendo cosas, yo no le voy a restringir algo que a él le va a favorecer. Una cosa es que se metiera en páginas que no debe, que estuviera usándola como para a lo mejor chatear con gente y... Insultar a gente o... Pero como yo sé que mi hijo está jugando a juegos, está viendo vídeos de los que él pueda aprender cosas. (Entrevista1, madre Jaime) 
No obstante, se trata de un uso vinculado fundamentalmente al entretenimiento. Resulta muy llamativo que no se haga uso de un ordenador para la realización de las tareas escolares encomendadas para casa y, en cambio, cuenten en el hogar con diversos dispositivos destinados al ocio (dos consolas, tres pantallas de televisión, dos tabletas, móviles, etc.). Consideramos que esta contraposición entre los deberes y el ocio es muy definitoria con respecto al capital cultural de la familia. La importancia que se le concede a los distintos dispositivos queda puesta de relieve al saber que disponen de un portátil que está averiado y no hay indicios de pretender solucionar el problema, mientras que recientemente se le rompió la tableta al niño y, por el contrario, sí se hace patente la intención de arreglarla.

\subsection{Normas y dispositivos}

El valor otorgado a las nuevas tecnologías tiene su correspondencia en el tiempo de uso dedicado y en las normas en el hogar que lo regulan. En el caso de la familia de capital cultural alto su uso para ocio -no para tareas escolares- se limita a los fines de semana, esta es la pauta que han establecido desde hace tiempo:

Madre: Pero bueno, la norma que siempre tuvimos en casa fue, únicamente el fin de semana ¿Vale? Para lo que es juego. Por la semana no. (Entrevista1, padres Catarina y Lucía)

Esta norma es perfectamente conocida y asumida por ambas hermanas, sin que ello conlleve conflictos por el tiempo de uso o por la demanda en algún otro momento no contemplado:

Catarina: Porque... Mis papás dicen que... Cuando era pequeña que... Sí, a ver, a no ser que estuviéramos enfermas, que lo utilicemos... O las, las consolas o algo digital, si es el ordenador... Para jugar me refiero, solo los fines de semanas o cuando sea vacaciones. Y ahora ya ellos, ya no nos lo tienen que decir, porque ya lo sabemos. (Entrevista1, Catarina)

El hecho de no disponer de mucho tiempo libre para emplear la tecnología con fines de ocio, dado que las niñas deben dedicar tiempo a las actividades escolares, pero también a su formación musical y a la práctica deportiva, resulta tranquilizador para los padres que no tienen que ejercer tanto control sobre su uso, al no existir ocasiones u oportunidades suficientes para que llegue a producirse un "abuso" potencial de los mismos.

Madre: Ni tampoco hay demasiado tiempo libre...

Padre: Tampoco.

Madre: Como para que puedan abusar de esas cosas... Está todo bastante... (Entrevista1, padres Catarina y Lucía)

En los hogares de capital cultural medio se establecen pautas de uso supeditadas a la realización de las tareas escolares y un cierto control paterno, pero donde los niños/as disponen de tiempo regulado para el ocio digital.

Madre: Efectivamente, entonces yo de lunes a viernes no les dejo utilizar ni la, ni la Wii, ni la Nintendo, ni en el ordenador. Solamente les dejo la tablet un poquito a la noche. Entonces, una vez que tienen todo hecho y tal, pues...

Entrevistador: ¿A la hora de cenar o así? 
Madre: Sí, se la dejo media horita. Habitualmente y cuando los otros también están conectados, entonces, juegan entre todos o hablan un poco, tal. Y después el fin de semana sí se la dejo, pero bueno, una hora a la mañana y otra a la tarde. Quiero decir... (Entrevista1, padres Alfonso y Antón)

Sin embargo, en el proceso de investigación se visualiza cierta laxitud con estas normas y los niños utilizaban estas tecnologías todo el tiempo. Si bien es cierto que los padres han incorporado una herramienta de control parental, éste se ocupa sustantivamente del tiempo que los niños están con los dispositivos. Y se han establecido normas (que muchas veces no se cumplen) sobre el uso de la tableta o los videojuegos, sólo en los fines de semanas. Las normas atañen a cuánto y cuándo pero no al contenido con el que están interactuando.

En el entorno familiar de nivel bajo, la tónica habitual se caracteriza por no fijar unos límites claros en el tiempo de uso de las TIC. A pesar de que madre e hijo apuntan que, de lunes a viernes, el tiempo dedicado al juego oscila entre 2 y 3 horas diarias, a lo largo de las entrevistas se constatan incoherencias que nos dan pie a pensar que podrían emplearse alrededor de 5 horas al día. Ya no sólo porque el niño tiene mucho tiempo libre -en parte, por la no participación en actividades extraescolares-, sino también por comentarios que dan a entender que juega hasta que se va a dormir: "A veces, cuando yo me voy para cama, él me juega [refiriéndose al padre]" (Entrevista2, Jaime), o el hecho de superar un juego en sólo tres días. Una situación que se intensifica los sábados y los domingos: "Los fines de semana yo diría que casi, casi todo el día" (Entrevista1, madre Jaime). Además, otro aspecto importante a destacar en relación con los videojuegos es que no existe ningún tipo de restricción sobre el uso de videojuegos violentos no recomendados para menores de 18 años.

La única regla parental que se cumple de manera tajante es la que atañe a la posesión del teléfono móvil. El niño no tendrá móvil propio hasta que la madre considere que cuenta con la responsabilidad necesaria para darle un uso correcto. Una norma que contrasta de lleno con la ausencia de indicaciones y restricciones, bien respecto al uso de la tableta y de las consolas, bien sobre el contenido de los videojuegos y los peligros que entraña Internet.

\section{Conclusiones}

Los hallazgos encontrados ponen de manifiesto el distinto significado social que las tecnologías pueden tener en el seno de la familia, y como éstas no son en ningún caso neutrales. Así, las diferencias relativas al capital cultural están muy presentes en las distintas formas de apropiación de la tecnología que se pone de manifiesto en el sentido que le otorgan y en las pautas que rigen su uso (Martínez Piñeiro, Gewerc Barujel \& Vila Couñago, 2018).

La competencia digital es vista como algo importante para familias de nivel medio y bajo, ya que lo entienden como un medio que favorece la movilidad social; poseer competencia tecnológica permitirá a sus hijos alcanzar una posición social más alta que la de la propia familia a través del acceso al mundo laboral y al contacto con otras personas y culturas que puede enriquecerles. En correspondencia con este valor otorgado, existe un escaso o nulo control sobre el uso de las tecnologías, especialmente en lo que atañe al contenido. 
En el caso de nivel alto privilegian otros entornos para el desarrollo de sus hijos e hijas, y la formación en otros ámbitos como el artístico o deportivo, aunque esto no conlleva que adquieran un nivel de competencia digital inferior al de muchos niños de su edad. En estos hogares las normas refuerzan al mismo tiempo la limitación del tiempo de uso de la tecnología para el ocio, sin que sea cuestionado por los menores. Esta pauta junto con la práctica de otras actividades más valoradas conduce a un tiempo reducido de uso de las tecnologías para este fin.

La apropiación de la competencia digital es, por lo tanto, no sólo un proceso material, sino al mismo tiempo simbólico, de dotación de sentido respecto a un determinado artefacto cultural por parte de un grupo social, donde los sujetos le otorgan significados de acuerdo a sus propios propósitos, y los incorporan en la vida cotidiana de los niños y en las dinámicas familiares (Winocur, 2007).

\section{Referencias}

Area, M. (2012). La alfabetización en la sociedad digital. En: M. Area, A. Gutiérrez, \& M. Á. Marzal, Alfabetización digital y competencias informacionales (pp. 3-42). Barcelona: Fundación Telefónica.

Bijker, W. E., \& Law, J. (Ed.). (1992). Shaping Technology / Building Society. London: The MIT Press.

Bourdieu, P. (2002). La distinción: Criterio y bases sociales del gusto. México: Taurus.

Buckingham, D. (2007). Digital Media Literacies: rethinking media education in the age of the Internet. Research in Comparative and International Education, 2(1), 43-55.

Colette, M. M., \& Da Silva, M. H. (2014). Novos Cenários de Aprendizagem, Inovação e Sustentabilidade: Uma Pesquisa-Ação na Graduação em Ciências e Tecnologia. RISTI: Revista Ibérica de Sistemas e Tecnologias de Informação, (E2), 55-69.

Creswell, J. W., \& Plano Clark, V. L. (2007). Designing and conducting mixed methods research. Thousand Oaks: Sage.

Da Cunha, A., da Silva Barbalho, M., de Rezende, L., \& Mourão Ferreira, R. (2015). O professor de Matemática do ensino médio e as tecnologias de informação e comunicação nas escolas públicas estaduais de Goiás. RISTI: Revista Ibérica de Sistemas e Tecnologias de Informação, (E4), 1-15.

Dussel, I. (2009). Los nuevos alfabetismos en el siglo XXI: Desafíos para la escuela. Recuperado de http://www.virtualeduca.info/Documentos/veBA09\%2O_ confDussel.pdf

Ferrari, A. (2013). DIGCOMP: A Framework for Developing and Understanding Digital Competence in Europe. Sevilla: Joint Research Centre, Institute for Prospective Technological Studies. doi: 10.2788/52966

Glaser, B. G., \& Strauss, A. L. (1967). The Discovery of grounded theory: strategies for qualitative research. New York: Aldine Publishing. 
Gutiérrez, A. B. (2005). Las prácticas sociales. Una introducción a Pierre Bourdieu. Córdoba, Argentina: Ferreyra.

Hollingworth, S., Mansaray, A., Allen, K., \& Rose, A. (2011) Parents' Perspectives on Technology and Children's Learning in the Home: Social Class and the Role of the Habitus. Journal of Computer Assisted Learning, 27(4), 347-360.

Johnson, N. F. (2009a). Cyber-relations in the Field of Home Computer Use for Leisure: Bourdieu and teenage technological experts. E-Learning, 6(2), 187-197.

Johnson, N. F. (2009b). Generational Differences in Beliefs about Technological Expertise. New Zealand Journal of Educational Studies, 44(1), 31-45.

Lankshear, C., \& Knobel, M. (2008). Digital Literacies: Concepts, Policies and Practices. New York: Peter Lang.

Livingston, S., \& Selfton-Green, J. (2016). The class. Living and learning in the digital age. New York: New York University press.

Martínez Piñeiro, E., Gewerc Barujel, A., \& Vila Couñago, E. (2018). La competencia digital de niños y niñas de doce años. Un estudio mixto secuencial explicativo. En: A. Pedro Costa, D. N. de Souza, P. A. de Castro, R. Arellano Saavedra, \& S. Oliveira e Sá (Eds.), Atas do $7^{\circ}$ Congresso Ibero-Americano em Investigaçao Qualitativa. Investigação Qualitativa na Educação. Volume I (pp. 233-242). Fortaleza, Brasil: Ludomedia.

Muñoz Justicia, J., \& Sahagún Padilla, M. (2017). Hacer análisis cualitativo con Atlas.ti 7. Manual de uso. Recuperado de http://manualatlas.psicologiasocial.eu/ atlasti7.pdf

North, S., Snyder, I., \& Bulfin, S. (2008). DIGITAL TASTES: Social class and young people's technology use. Information, communication \& society, 11(7), 895-911.

OCDE (2014). Education at a Glance 2014. OECD indicators. OECD Publishing. Recuperado de http://dx.doi.org/10.1787/eag-2014-en

Soãres de Lima, S. B., Luzia Leite, J., Lorenzini Erdmann, A., Giacomelli Prochnow, A., Conceição Stipp, M. A., \& Real Lima García, V. R. (2010). La Teoría Fundamentada en Datos. Un camino a la investigación en enfermería. Index de Enfermería, 19(1), 55-59. doi: 10.4321/S1132-12962010000100012

Valcke, M., Bonte, S., Wever, B., \& Rots, I. (2010). Internet Parenting Styles and the Impact on Internet Use of Primary School Children. Computers \& Education, 55(2), 454-464. doi: 10.1016/j.compedu.2010.02.009

Van Dijk, J. \& Van Deursen, A. (2014). Digital skills: unlocking the information society (1 ${ }^{\text {a }}$ Ed.). New York, NY: Palgrave Macmillan. 
Vuorikari, R., Punie, Y., Carretero, S., \& Van den Brande, L. (2016). DigComp 2.o: The Digital Competence Framework for Citizens. Update Phase 1: The Conceptual Reference Model. European Comission. Recuperado de http://publications.jrc. ec.europa.eu/repository/bitstream/JRC101254/jrc101254_digcomp\%202.0\%20 the\%20digital\%20competence\%2oframework\%2ofor\%20citizens.\%2oupdate\%20 phase\%201.pdf

Winocur, R. (2007). Nuevas tecnologías y usuarios. La apropiación de las TIC en la vida cotidiana. Telos: Cuadernos de Comunicación, Tecnología y Sociedad, 73. Recuperado de https://telos.fundaciontelefonica.com/telos/articuloexperiencia. asp@idarticulo=1\&rev=73.htm

Yin, R. K. (2003). Case study research. Design and methods ( $3^{\text {a }}$ Ed.). Londres: Sage. 